\title{
Improvement of Outcomes in Hemodynamically Unstable Patients With Pelvic Fracture Following The Establishment of a Trauma Center: A Single Center Observational Study in Korea
}

Mina Lee

Gachon University

Byungchul Yu ( $\square$ kane2123@gilhospital.com )

Gachon University https://orcid.org/0000-0002-2444-2343

Giljae Lee

Gachon University

Jungnam Lee

Gachon University

Kangkook Choi

Gachon University

Youngeun Park

Gachon University

Jihun Gwak

Gachon University

Research article

Keywords: Pelvic, Trauma, Trauma center, Pre-peritoneal pelvic packing, REBOA

Posted Date: January 6th, 2021

DOI: https://doi.org/10.21203/rs.3.rs-139385/v1

License: (c) (i) This work is licensed under a Creative Commons Attribution 4.0 International License. Read Full License 


\section{Abstract}

Background: The trauma center and multidisciplinary management protocols have been proved to improve the outcomes of severely injured patients. Hemorrhage from pelvic injury is associated with high mortality and is a common cause of preventable trauma death. This study aims to evaluate the effects of the establishment of a trauma center and management protocols on the outcomes of hemodynamically unstable patients with pelvic fractures.

Methods: Hemodynamically unstable patients with pelvic fractures were reviewed retrospectively over a 10 -year period. They were grouped into periods 1 and 2, which were defined as before and after the establishment of a trauma center and protocols, respectively. Basic characteristics and outcomes were compared between periods.

Results: This study enrolled a total of 106 patients. Basic and physiological characteristics were not significantly different in both periods. Pre-peritoneal packing (PPP) and resuscitative endovascular balloon occlusion of aorta (REBOA) were only performed in period 2 (PPP, $N=27$; REBOA, $N=10$ ). In period 2, the time from emergency room arrival to hemostatic intervention was significantly shorter (269 \pm $132.4 \mathrm{~min}$. vs. $147.2 \pm 95.5 \mathrm{~min}$., $\mathrm{p}<0.0001$ ), and mortality due to acute hemorrhage was significantly decreased $(33.3 \%$ vs. $8.6 \%, p=0.018)$. The multivariate logistic regression analysis identified age, ISS, and period 1 as independent risk factors for mortality.

Conclusion: The establishment of a trauma center and multidisciplinary management protocols, such as PPP and REBOA, improved the outcomes of hemodynamically unstable patients with pelvic fractures.

\section{Introduction}

Trauma is a leading cause of death for young people in Korea. The rate of preventable deaths is reportedly decreasing but still higher compared to those in developed countries. $(1,2)$ Therefore, the Ministry of Health and Welfare and medical professionals decided to establish regional trauma centers in 2012. The first regional trauma center opened in 2014, with plans to establish a total of 17 regional trauma centers in Korea by 2021. Several changes may be observed after the establishment of a trauma center, such as an increase in the volume of trauma cases in the center, trauma-dedicated resources, performance improvement programs, or the development of a regional trauma system.

The mortality rate of hemodynamically unstable patients with pelvic fractures remains greater than $30 \%$ despite advancements in the management of severely injured patients.(3-6) Furthermore, it is also one of the most frequent contributors to traumatic preventable death.(7) Therefore, management strategies for these patients including initial resuscitation, diagnosis, and therapeutic interventions are essential in reducing mortality. The majority of trauma centers emphasize angioembolization (AE) as an effective intervention to stop pelvic bleeding, although $A E$ cannot address the venous or bony hemorrhage within the pelvis. $(8,9)$ However, the utilization of $A E$ needs a proper facility, equipment, and human resources. It 
is well known that time to hemorrhage control is related to mortality, but it typically takes around 2-5 hours before $\mathrm{AE}$ is performed in many centers. $(5,6,10,11)$

Pre-peritoneal pelvic packing (PPP) with external pelvic compression reportedly stops hemorrhage and facilitates other emergent operative procedures. $(9,12)$ We included PPP and pelvic circumferential compression devices in the pelvic fracture management algorithm after the trauma center was established in 2014 and added resuscitative endovascular balloon occlusion of aorta (REBOA) to the management algorithm in 2016.(13) The aim of this study was to evaluate the time to intervention and the outcomes of hemodynamically unstable patients with pelvic fractures before and after the establishment of a trauma center and management protocol. We hypothesized the establishment of a trauma center, and protocols including interventions such as PPP and REBOA will shorten the time to intervention and lower mortality in our institution.

\section{Materials And Methods}

\section{Setting}

The Gachon University Gil Medical Center is a 1,500-bed teaching hospital in Incheon, South Korea that serves a population of 3 million people. The regional trauma center was established in 2014 in accordance with the trauma system development plan of the Ministry of Health and Welfare. Before 2014 , the emergency center managed trauma patients without a dedicated trauma surgeon, trauma team, dedicated facilities, or protocols for the evaluation and management of pelvic injury. There were more than 3,000 trauma-related admissions, of which 500-550 patients presented with major trauma (injury severity score $>15$ ). The regional trauma center is equipped with a trauma bay, 2 operating rooms dedicated to trauma, a 20-bed trauma intensive care unit, and a trauma angiography suite. Fourteen fulltime trauma surgeons currently work at the center, and a team of emergency physicians, anesthesiologists, and orthopedic and neurosurgery consultants are available 24/7.

\section{Study population}

The study included hemodynamically unstable patients with pelvic fractures from January 2009 to December 2018. Hemodynamic instability was defined as having a systolic blood pressure (SBP) $<90$ $\mathrm{mmHg}$ during the initial resuscitation period despite the transfusion of 2 units of packed red blood cells (pRBCs). We excluded patients who died in the emergency room (ER) or from out-of-hospital cardiac arrest, patients $<16$ years old, patients without interventions to control pelvic bleeding, and those who received delayed interventions after treatment of other injuries. The outcomes of the enrolled patients were analyzed by time period; periods 1 and 2 were defined as before and after, respectively, establishment of the trauma center and protocols.

\section{Protocol}


During period 1, patients with pelvic injuries were initially evaluated and managed at the ER by emergency physicians. After evaluation and initial resuscitation, the general and orthopedic surgeons on call decided on further management plans. Angioembolization (AE) was mainly performed if extravasation of contrast was seen on computed tomography (CT). Pelvic circumferential compression devices or external fixations were not utilized in this period. There was no protocol for the management of bleeding pelvic fracture patients.

During period 2, severely injured patients were managed at the trauma bay by a trauma team utilizing dedicated facilities. Patients with bleeding pelvic fractures were managed according to a previously published algorithm (Fig. 1). Patients who tested positive for abdominal fluids on FAST were brought to the operating room for both laparotomy and pre-peritoneal packing (PPP). External fixation was performed following the operations, if available. If the patients remained hemodynamically unstable despite PPP, AE was then performed. For patients with negative FAST results, AE was attempted first. However, if $A E$ was not immediately available, or the patient was hemodynamically unstable while waiting for AE, PPP was performed as a first choice. Since 2016, we implemented REBOA in the algorithm for extremely unstable patients.

\section{Statistical analysis}

Numerical data were presented as the means and standard deviations, whereas categorical data were presented as percentages. The univariate analysis was performed using the Student's $t$ test and chisquared test. Covariables with significance in the univariate analysis were analyzed in a multivariate regression model. Multivariable logistic regression was utilized to evaluate independent risk factors for mortality. Statistical significance was defined as $p<0.005$. All statistical analyses were performed using SPSS version 20.0 (IBM Corp., Armonk, NY, USA)

\section{Results}

We extracted data from 1,469 patients with an AIS pelvis score over 1 point from the trauma center database. A total of 106 (7.2\%) patients met the inclusion criteria of the current study (Fig. 2). The mean age of patients was $52.3 \pm 19.8$ years, and $53(50 \%)$ of the patients were male. Demographic data indicated a mean SBP at ER admission of $83.5 \pm 29.2$, the lowest SBP at ER of $60.4 \pm 25.7$, a Glasgow Coma Scale (GCS) of $12 \pm 4.2$, and ISS of $39 \pm 11$.3. The basic demographics of the two groups were statistically similar (Table 1). The mean amounts of pRBC and fresh frozen plasma (FFP) transfusion for the first 24 hours were $16.4 \pm 13.8$ and $10.6 \pm 10.4$, respectively. The mean amount of FFP transfusion for 24 hours was significantly increased from $7.5 \pm 4.9$ to $12.2 \pm 12.1$ in period $2(p=0.028)$. The mean time to intervention (for PPP, $A E$, or both) was $269 \pm 132.4$ minutes in period 1 and $147.2 \pm 95.5$ minutes in period $2(p<0.001)$. The mean time to AE only was also significantly shorter in period $2(p=0.002)$. The time to intervention decreased throughout the years (Fig. 3), and REBOA was performed only in period 2 for 10 patients (Table 2). Patients who underwent REBOA had lower SBP and higher lactate and ISS 
compared to those who did not. In 8 patients, REBOA balloon was deployed in Zone 3. Seven patients survived for more than 24 hours and 3 patients were able to survive and get discharged (Table 3 ).

The overall mortality rate of the study population was $34.9 \%$; from $47.2 \%$ in period 1 , this decreased to $28.6 \%$ in period $2(p=0.056)$. The mortality rate associated with acute hemorrhagic shock also decreased from $33.3 \%$ in period 1 to $8.6 \%$ in period 2 , and it was statistically significant $(p=0.018)$. There were no TRISS unexpected survivors (TRISS probability of survival < 0.5 ) in period 1, but there were $10(24 \%)$ unexpected survivors in period 2 (Table 2). Patients who survived were found to be significantly younger ( $49.6 \pm 20.1$ vs. $57.4 \pm 18.6, p=0.046)$ and have higher SBP $(66.8 \pm 23.6$ vs. $48.4 \pm 25.4, p=0.001), G C S$, and TRISS, with lower ISS, lactate, base deficit, and transfusion amounts $(p<0.001)($ Table 4$)$.

A multivariate logistic regression analysis evaluated the independent risk factors for mortality as listed in Table 5. After adjusting for age, sex, base deficit, lactate, ISS, TRISS, and transfusion amounts, the following variables were found to be significantly associated with overall mortality: age (odds ratio, 1.19; $95 \%$ confidence interval [Cl], 1.04-1.35), ISS (odds ratio, 1.16; 95\% confidence interval [Cl], 1.02-1.32), and period 1 (odds ratio, $13.4 ; 95 \%$ confidence interval [Cl], 3.02-59.89).

\section{Discussion}

Trauma center establishment has been associated with reduced mortality in several studies, and it is mainly due to increased annual trauma volume, trauma-dedicated resources, and performance improvement program (PIP).(14-16) Before the establishment of a regional trauma center, approximately 250 severely injured patients (ISS > 15) were admitted to our hospital annually. However, after the trauma center opened in 2014, more than 500 severely injured patients were managed annually. The volume of trauma patients and the rate of direct transportation increase year by year.(17) Furthermore, the presence of human resources (trauma-dedicated surgeons, nurses, and a trauma program manager) and a PIP could influence resuscitation practice, time to intervention, and hemorrhage control modalities.(8)

Bleeding pelvic fractures are uncommon but are associated with significant mortality due to hemorrhagic shock. Before the establishment of a trauma center, hemodynamically unstable patients with pelvic fractures were managed mainly by an emergency physician at the initial resuscitation phase. After diagnosis of bleeding pelvic fracture was confirmed using imaging studies, general and orthopedic surgical services were contacted. In many cases, however, there were conflicts between departments when deciding on further treatment interventions. In one study, the application of a multidisciplinary clinical pathway with joint decision making between trauma and orthopedic surgeons resulted in improved outcomes in patients with pelvic fractures.(14) Therefore, rapid hemostasis with appropriate treatment following a multidisciplinary algorithm involving hemostatic interventions is crucial. We implemented this pelvic fracture management protocol in 2014 shortly after the trauma center establishment.

The ATLS guidelines recommend that a pelvic binder (PB) should be used for the external compression of pelvic cavity when there are signs of a pelvic ring fracture.(18) Studies have also demonstrated that 
commercial PBs are more effective in controlling pelvic bleeding than a conventional "bed-sheet" compression.(19) In Korea, prehospital PB application is very rare, and our hospital used the conventional "bed-sheet" compression in a few cases until 2014. After the official approval of commercial PBs by the Ministry of Food and Drug Safety in 2013, we used these in the management algorithm for suspected pelvic fracture patients in 2014. External pelvic fixations are rarely used in this study because the orthopedic surgeons in our hospital prefer PB application over these. In a study comparing PB and external fixation in patients with pelvic fractures, those who underwent external fixation needed higher amounts of transfusion.(20) Thus, until now, we mainly utilize PB for external compression purposes in the algorithm for pelvic injury management.

AE has been used since the 1970s for hemorrhage control in patients with pelvic bone fractures. It has been proved to be effective and remains the most widely used hemostatic intervention. However, AE may not be an effective intervention to stop bleeding due to pelvic fracture, because approximately $80 \%$ of the bleeding is associated with bone or venous bleeding.(9) Venous bleeding cannot be managed by $A E$, so $\mathrm{AE}$ in hemodynamically unstable pelvic injuries results in poor patient outcomes with mortality rates greater than $40 \%$.(9) In addition, the availability of $A E$ varies by institution. AE requires specific facilities, equipment, and human resources, so some centers are unable to perform this technique. Even in centers that can perform $A E$, it might not be available immediately, specifically during nights or weekends, which can further increase the mortality rate. $(11,21)$ Several studies have reported that the time from ER arrival to AE takes at least 2 hours up to more than 5 hours. $(22,23)$ In our study, the time to AE only decreased from 269 minutes in period 1 to 181 minutes in period 2, but this waiting time is still too long for hemodynamically unstable patients.

PPP has been proposed as an alternative intervention to AE. PPP is a simple procedure, so it can be performed in the resuscitation room or operation room by a trauma surgeon within an hour of patient arrival. The time to PPP was 44-55 minutes in recent studies, and this is significantly shorter compared to AE. $(5,24)$ PPP could significantly decrease the time to intervention in our study also. Studies demonstrated that an implementation of clinical guidelines that include PPP for hemodynamically unstable patients led to a significant decrease in transfused blood products and in mortality. $(5,24)$ More recent studies have revealed that PPP is a safe and rapid intervention associated with significantly reduced mortality in hemodynamically unstable patients with pelvic fractures compared to patients managed by conventional intervention without PPP. $(25,26)$ Moreover, unstable pelvic fractures are often associated with intra-abdominal injuries. One study reported that a total of $34.3 \%$ of severe pelvic fracture patients had associated intra-abdominal injuries including bladder and bowel injuries.(27) In such cases, AE might delay abdominal exploration, or a laparotomy might delay hemostasis using AE. However, a midline laparotomy and PPP can be performed simultaneously with separate incisions, so it achieves hemorrhage and contamination control rapidly without concerns of cross-contamination in the retroperitoneal space from bowel injuries.(12) In this study, concomitant laparotomy increased in period 2 after inclusion of PPP in the management algorithm. In addition, laparotomy for the decompression of abdominal pressure in patients with suspected abdominal compartment syndrome was only performed in period 2. The World Society of Emergency Surgery (WSES) guidelines for pelvic fracture recommend that 
PPP should always be considered in hospitals with no AE services and that PPP is an effective surgical measure for early hemorrhage control in hypotensive patients with bleeding pelvic disruptions.(18) Therefore, PPP is recommended as a first intervention for hemodynamically unstable patients (WSES grade IV) in the pelvic trauma management algorithm.(18)

REBOA has recently been reported as a viable alternative to performing an aortic cross clamp (ACC) through emergent thoracotomy. $(28,29)$ REBOA can be a "bridge" procedure in torso hemorrhages followed by an operation or AE. REBOA can be placed in Zone 1 (supra-celiac) or Zone 3 (infra-renal). Zone 3 REBOA can be optimal, especially for pelvic bleeding, because it can raise blood pressure and reduce arterial bleeding associated with pelvic injury while preventing ischemic insult on visceral organs on top of having a long occlusion time.(30) Although there is little evidence of mortality benefit in previous articles, the WSES guidelines suggest REBOA as an alternative to ACC and that Zone 3 REBOA should be considered to be a bridge to definitive treatment in hemodynamically unstable patients.(18) Our center has implemented REBOA in the pelvic injury management algorithm since 2016, and REBOA was performed in 10 patients; $80 \%$ of these were deployed in Zone 3 with 3 patients surviving and getting discharged.

The purpose of this study was to evaluate changes in outcomes after trauma center establishment and pelvic injury management protocol including PPP and REBOA. This study demonstrated significantly decreased time to interventions and mortality due to acute hemorrhage. We believe that these changes are due to (1) an attending trauma surgeon being a leader of multidisciplinary team; (2) management protocols including PB, PPP, and REBOA; (3) an increase in concomitant laparotomy; and (4) changes in blood product usage. We think these changes might not be achieved without the establishment of a trauma center with financial support in developing countries such as Korea.

This study has several limitations. The main limitations are its retrospective study design and small sample size due to the study being only from a single institute. These might have introduced bias. In addition, we investigated in-hospital mortality only, and these might have influenced our results. Finally, we could not evaluate the adherence of individual surgeons to the management algorithm. In the future, a well-designed multi-center prospective study with a larger sample size should be designed to analyze the relationship between trauma center establishment and patient outcomes.

\section{Conclusion}

The improved outcomes in hemodynamically unstable patients with pelvic fracture were achieved because of the establishment of a trauma center and multidisciplinary management protocols. Trauma center designation can advance the management of severe trauma cases such as pelvic fracture, especially in developing countries.

\section{Abbreviations}


ACC: Aortic Cross Clamping; AE: Angioembolization; CT: Computed Tomography; ER: Emergency room; FFP: Fresh Frozen Plasma; GCS: Glasgow Coma Scale; ISS: Injury Severity Score; PPP: Pre-peritoneal Pelvic Packing; pRBC: Packed Red Blood Cell; PIP: Performance Improvement Program; PB: Pelvic Binder; REBOA: Resuscitative Endovascular Balloon Occlusion of Aorta; SBP: Systolic Blood Pressure (SBP); TRISS: Trauma Injury Severity Score

\section{Declarations}

\section{Acknowledgements}

Not apllicable

\section{Funding}

The author(s) received no financial support for the research, authorship, and/or publication of this article.

\section{Availability of data and materials}

Data and materials can be made available to the public through direct requests to the corresponding author.

\section{Authors' contributions}

MAL and BCY had full access to all the data and drafted the manuscript. JNL, GJL, YEP, JHG and KKC participated in the study design and the education.

\section{Ethical approval and consent to participate}

This study was reviewed and approved by Gachon University Ethics Committee (approval number: 2020471).

\section{Consent for publication}

All authors agree with the publication of this article

\section{Competing interests}

The author(s) declare no competing interests with respect to the research, authorship, and/or publication of this article.

\section{References}

1. S. K. 2018 Result on the cause of death statistics. Statistics Korea. 2018.

2. Kim H, Jung KY, Kim SP, Kim SH, Noh H, Jang HY, et al. Changes in preventable death rates and traumatic care systems in Korea. J Korean Soc Emerg Med. 2012;23(2):189-97.

3. Stein D, O'toole R, Scalea T. Multidisciplinary approach for patients with pelvic fractures and hemodynamic instability. Scand J Surg. 2007;96(4):272-80. 
4. White CE, Hsu JR, Holcomb JB. Haemodynamically unstable pelvic fractures. Injury. 2009;40(10):1023-30.

5. Jang JY, Shim H, Jung PY, Kim S, Bae KS. Preperitoneal pelvic packing in patients with hemodynamic instability due to severe pelvic fracture: early experience in a Korean trauma center. Scand J Trauma Resusc Emerg Med. 2016;24(1):1-7.

6. Lee MA, Yu B, Lee J, Park JJ, Lee GJ, Choi KK, et al. Effects of the establishment of a trauma center and a new protocol on patients with hemodynamically unstable pelvic fractures at a single institution in Korea. Eur J Trauma Emerg Surg. 2019;45(2):273-9.

7. Jung K, Kim I, Park SK, Cho H, Park CY, Yun J-H, et al. Preventable trauma death rate after establishing a national trauma system in Korea. J Korean Med Sci. 2019;34(8).

8. Agolini SF, Shah K, Jaffe J, Newcomb J, Rhodes M, Reed JF. Arterial embolization is a rapid and effective technique for controlling pelvic fracture hemorrhage. J Trauma Acute Care Surg. 1997;43(3):395-9.

9. Cothren CC, Osborn PM, Moore EE, Morgan SJ, Johnson JL, Smith WR. Preperitonal pelvic packing for hemodynamically unstable pelvic fractures: a paradigm shift. J Trauma Acute Care Surg. 2007;62(4):834-42.

10. Tesoriero RB, Bruns BR, Narayan M, Dubose J, Guliani SS, Brenner ML, et al. Angiographic embolization for hemorrhage following pelvic fracture: is it "time" for a paradigm shift? J Trauma Acute Care Surg. 2017;82(1):18-26.

11. Schwartz DA, Medina M, Cotton BA, Rahbar E, Wade CE, Cohen AM, et al. Are we delivering two standards of care for pelvic trauma? Availability of angioembolization after hours and on weekends increases time to therapeutic intervention. J Trauma Acute Care Surg. 2014;76(1):134-9.

12. Burlew CC, Moore EE, Smith WR, Johnson JL, Biffl WL, Barnett CC, et al. Preperitoneal pelvic packing/external fixation with secondary angioembolization: optimal care for life-threatening hemorrhage from unstable pelvic fractures. J Am Coll Surg. 2011;212(4):628-35.

13. Biffl WL, Fox CJ, Moore EE. The role of REBOA in the control of exsanguinating torso hemorrhage. $J$ Trauma Acute Care Surg. 2015;78(5):1054-8.

14. Boyd CR, Tolson MA, Copes WS. Evaluating trauma care: the TRISS method. J Trauma Acute Care Surg. 1987;27(4):370-8.

15. Sampalis JS, Denis R, Frechette P, Brown R, Fleiszer D, Mulder D. Direct transport to tertiary trauma centers versus transfer from lower level facilities: impact on mortality and morbidity among patients with major trauma. J Trauma Acute Care Surg. 1997;43(2):288-96.

16. Halm EA, Lee C, Chassin MR. Is volume related to outcome in health care? A systematic review and methodologic critique of the literature. Ann Intern Med. 2002;137(6):511-20.

17. Yu B, Lee MA, Hyun S, Yoon Y-c. Trauma volume and performance of a regional trauma center in Korea: initial 5-year analysis. J Trauma Inj. 2020;33(1):31-7.

18. Coccolini F, Stahel PF, Montori G, Biffl W, Horer TM, Catena F, et al. Pelvic trauma: WSES classification and guidelines. World J Emerg Surg. 2017;12(1):1-18. 
19. Tan EC, van Stigt SF, van Vugt AB. Effect of a new pelvic stabilizer (T-POD $\left.{ }^{8}\right)$ on reduction of pelvic volume and haemodynamic stability in unstable pelvic fractures. Injury. 2010;41(12):1239-43.

20. Krieg JC, Mohr M, Ellis TJ, Simpson TS, Madey SM, Bottlang M. Emergent stabilization of pelvic ring injuries by controlled circumferential compression: a clinical trial. J Trauma Acute Care Surg. 2005;59(3):659-64.

21. Tanizaki S, Maeda S, Matano H, Sera M, Nagai H, Ishida H. Time to pelvic embolization for hemodynamically unstable pelvic fractures may affect the survival for delays up to $60 \mathrm{~min}$. Injury. 2014;45(4):738-41.

22. Gänsslen A, Giannoudis $P$, Pape H-C. Hemorrhage in pelvic fracture: who needs angiography? Curr Opin Crit Care. 2003;9(6):515-23.

23. Osborn PM, Smith WR, Moore EE, Cothren CC, Morgan SJ, Williams AE, et al. Direct retroperitoneal pelvic packing versus pelvic angiography: a comparison of two management protocols for haemodynamically unstable pelvic fractures. Injury. 2009;40(1):54-60.

24. Burlew CC, Moore EE, Stahel PF, Geddes AE, Wagenaar AE, Pieracci FM, et al. Preperitoneal pelvic packing reduces mortality in patients with life-threatening hemorrhage due to unstable pelvic fractures. J Trauma Acute Care Surg. 2017;82(2):233.

25. Chiara O, di Fratta E, Mariani A, Michaela B, Prestini L, Sammartano F, et al. Efficacy of extraperitoneal pelvic packing in hemodynamically unstable pelvic fractures, a propensity score analysis. World J Emerg Surg. 2016;11(1):22.

26. Li Q, Dong J, Yang Y, Wang G, Wang Y, Liu P, et al. Retroperitoneal packing or angioembolization for haemorrhage control of pelvic fractures-quasi-randomized clinical trial of 56 haemodynamically unstable patients with Injury Severity Score $\geq 33$. Injury. 2016;47(2):395-401.

27. Cho J, Benjamin E, Inaba K, Lam L, Demetriades D. Severe bleeding in pelvic fractures: considerations in planning damage control. Am Surg. 2018;84(2):267-72.

28. Stannard A, Eliason JL, Rasmussen TE. Resuscitative endovascular balloon occlusion of the aorta (REBOA) as an adjunct for hemorrhagic shock. J Trauma. 2011;71(6):1869-72.

29. DuBose JJ, Scalea TM, Brenner M, Skiada D, Inaba K, Cannon J, et al. The AAST prospective Aortic Occlusion for Resuscitation in Trauma and Acute Care Surgery (AORTA) registry: data on contemporary utilization and outcomes of aortic occlusion and resuscitative balloon occlusion of the aorta (REBOA). J Trauma Acute Care Surg. 2016;81(3):409-19.

30. Coccolini F, Ceresoli M, McGreevy DT, Sadeghi M, Pirouzram A, Toivola A, et al. Aortic balloon occlusion (REBOA) in pelvic ring injuries: preliminary results of the $A B O$ Trauma Registry. Updates Surg. 2020:1-10.

\section{Tables}

Table 1. General characteristics of all patients managed in the period 1 and period 2 


\begin{tabular}{|lllll|}
\hline & $\begin{array}{l}\text { Total } \\
(\mathrm{N}=106)\end{array}$ & Period 1 (N=36) & Period 2 (N=70) & $p$ value \\
\hline Age & $52.3 \pm 19.8$ & $51.2 \pm 19.2$ & $52.9 \pm 20.3$ & 0.667 \\
\hline Sex, male (\%) & $53(50)$ & $18(50)$ & $35(50)$ & 1 \\
\hline SBP upon ED arrival (mmHg) & $83.5 \pm 29.2$ & $87.9 \pm 25.5$ & $81.2 \pm 30.9$ & 0.231 \\
\hline Lowest SBP at ED (mmHg) & $60.4 \pm 25.7$ & $59.9 \pm 25$ & $60.6 \pm 26.2$ & 0.894 \\
\hline Hemoglobin (mg/dL) & $10.9 \pm 2.7$ & $10.5 \pm 2.2$ & $11.1 \pm 2.8$ & 0.206 \\
\hline INR & $1.71 \pm 2.15$ & $1.86 \pm 2.45$ & $1.65 \pm 2$ & 0.658 \\
\hline Lactate (mmol/L) & $4.84 \pm 3.19$ & $4.26 \pm 2.73$ & $5.13 \pm 3.37$ & 0.161 \\
\hline Base deficit (mmol/L) & $8.41 \pm 5.81$ & $7.93 \pm 5.73$ & $8.66 \pm 5.89$ & 0.546 \\
\hline GCS & $12 \pm 4.2$ & $12.3 \pm 3.9$ & $11.8 \pm 4.1$ & 0.559 \\
\hline ISS & $39 \pm 11.3$ & $38.1 \pm 12.3$ & $39.5 \pm 10.9$ & 0.55 \\
\hline TRISS & $0.62 \pm 0.32$ & $0.67 \pm 0.31$ & $0.6 \pm 0.32$ & 0.267 \\
\hline
\end{tabular}

SBP: systolic blood pressure, ED: emergency department, INR: international normalized ratio, GCS: Glasgow coma scale, ISS: Injury severity score, TRISS: trauma and injury severity score

Table 2 Intervention related data and outcomes for each period 


\begin{tabular}{|lllll|}
\hline & $\begin{array}{l}\text { Total } \\
(\mathbf{N}=106)\end{array}$ & $\begin{array}{l}\text { Period 1 } \\
(\mathbf{N}=36)\end{array}$ & $\begin{array}{l}\text { Period 2 } \\
(\mathbf{N}=70)\end{array}$ & p value \\
\hline Time to intervention (minutes) & $\begin{array}{l}188.9 \pm \\
123.4\end{array}$ & $269 \pm 132.4$ & $147.2 \pm 95.5$ & $<0.0001$ \\
\hline Time to AE only, N=79 & $\begin{array}{l}221.6 \pm \\
123.5\end{array}$ & $269 \pm 132.4$ & $181.9 \pm 100.9$ & 0.002 \\
\hline Time to PPP only, N=6 & $83.5 \pm 30.5$ & & $83.5 \pm 30.5$ & \\
\hline Time to AE + PPP, N=21 & $91.6 \pm 51.2$ & & $91.6 \pm 51.2$ & \\
\hline Laparotomy, n(\%) & $15(14.2)$ & $1(2.8)$ & $14(20)$ & 0.012 \\
\hline REBOA, $\mathbf{n}$ (\%) & 10 & 0 & $10(14.3)$ & \\
\hline pRBC for the first 4 hours (units) & $7.5 \pm 6.3$ & $7.1 \pm 5.3$ & $7.6 \pm 6.9$ & 0.644 \\
\hline FFP for the first 4 hours (units) & $3.3 \pm 3.2$ & $3.3 \pm 2.7$ & $3.3 \pm 3.4$ & 0.917 \\
\hline pRBC for the first 24 hours (units) & $16.4 \pm 13.8$ & $13.1 \pm 9.6$ & $18.1 \pm 15.4$ & 0.08 \\
\hline FFP for the first 24 hours (units) & $10.6 \pm 10.4$ & $7.5 \pm 4.9$ & $12.2 \pm 12.1$ & 0.028 \\
\hline Overall mortality, N (\%) & $37(34.9)$ & $17(47.2)$ & $20(28.6)$ & 0.056 \\
\hline 24-hour mortality, N(\%) & $18(17)$ & $9(25)$ & $9(12.9)$ & 0.143 \\
\hline 30-day mortality, N(\%) & $19(17.9)$ & $8(22.2)$ & $11(15.7)$ & 0.149 \\
\hline $\begin{array}{l}\text { Mortality due to acute hemorrhage, } \\
\text { N(\%) }\end{array}$ & $17(16)$ & $10(33.3)$ & $6(8.6)$ & 0.018 \\
\hline Unexpected survivor*, N & 10 & 0 & 10 & 0.03 \\
\hline
\end{tabular}

AE: angioembolization, PPP: pre-peritoneal packing, REBOA: resuscitative endovascular balloon occlusion of aorta, pRBC: packed red blood cell, FFP: fresh frozen plasma

*Unexpected survivor: Number of observed survivor among patients whose TRISS probability of survival < 0.5

Table 3 Characteristics and outcomes of patients underwent REBOA 


\begin{tabular}{|ll|}
\hline & REBOA group (N=10) \\
\hline Age & $54.9 \pm 18.4$ \\
\hline SBP upon ED arrival (mmHg) & $65.3 \pm 18.3$ \\
\hline Lowest SBP at ED (mmHg) & $50.5 \pm 7.2$ \\
\hline Lactate (mmol/L) & $7.86 \pm 3.12$ \\
\hline ISS & $43.6 \pm 10.4$ \\
\hline REBOA deployment in Zone 3, N (\%) & $8(80)$ \\
\hline Death, N (\%) & $7(70)$ \\
\hline Death in 24 hours, N (\%) & $3(30)$ \\
\hline Death in 30 days, N (\%) & $4(40)$ \\
\hline
\end{tabular}

REBOA: resuscitative endovascular balloon occlusion of aorta, SBP: systolic blood pressure, ISS: Injury severity score

Table 4 Comparison of the characteristic between survivor and non-survivor

\begin{tabular}{|llll|}
\hline & Survivor, N=69 & Non-survivor, N=37 & $p$ value \\
\hline Age & $49.6 \pm 20.1$ & $57.4 \pm 18.6$ & 0.046 \\
\hline SBP upon ED arrival (mmHg) & $87.5 \pm 27.8$ & $75.9 \pm 30.7$ & 0.59 \\
\hline Lowest SBP at ED (mmHg) & $66.8 \pm 23.6$ & $48.4 \pm 25.4$ & 0.001 \\
\hline Lactate (mmol/L) & $4.05 \pm 2.54$ & $6.28 \pm 3.73$ & $<0.0001$ \\
\hline Base deficit (mmol/L) & $7.29 \pm 4.6$ & $10.6 \pm 7$ & 0.004 \\
\hline GCS & $13.5 \pm 2.8$ & $9.1 \pm 4.8$ & $<0.0001$ \\
\hline ISS & $36.3 \pm 10.4$ & $44.1 \pm 11.3$ & 0.001 \\
\hline TRISS & $0.733 \pm 0.028$ & $0.417 \pm 0.057$ & $<0.0001$ \\
\hline pRBC for the first 4 hours (units) & $5.3 \pm 4.3$ & $11.4 \pm 7.7$ & $<0.0001$ \\
\hline FFP for the first 4 hours (units) & $2.4 \pm 2.4$ & $4.9 \pm 3.8$ & $<0.0001$ \\
\hline pRBC for the first 24 hours (units) & $11.8 \pm 9.6$ & $25.1 \pm 16.4$ & $<0.0001$ \\
\hline FFP for the first 24 hours (units) & $11.8 \pm 9.6$ & $16.2 \pm 12.9$ & $<0.0001$ \\
\hline Period 1, N(\%) & $19(27.5)$ & $17(45.8)$ & 0.056 \\
\hline
\end{tabular}


SBP: systolic blood pressure, ED: emergency department, GCS: Glasgow coma scale, ISS: Injury severity score, TRISS: trauma and injury severity score, pRBC: packed red blood cell, FFP: fresh frozen plasma

Table 5 Logistic regression analysis of the independent risk factors of mortality

\begin{tabular}{|lllll|}
\hline & \multicolumn{2}{c}{$\boldsymbol{p \text { value }}$} & Odds ratio to mortality & \multicolumn{2}{c|}{$\mathbf{9 5 \%}$ confidence interval } \\
\cline { 4 - 5 } & & & Lower & Upper \\
\hline Period 1 & 0.001 & 13.442 & 3.017 & 59.895 \\
\hline Age & 0.011 & 1.185 & 1.040 & 1.351 \\
\hline Base deficit & 0.775 & 0.973 & 0.808 & 1.172 \\
\hline Lactate & 0.492 & 1.121 & 0.809 & 1.553 \\
\hline ISS & 0.023 & 1.162 & 1.021 & 1.324 \\
\hline
\end{tabular}

Figures 


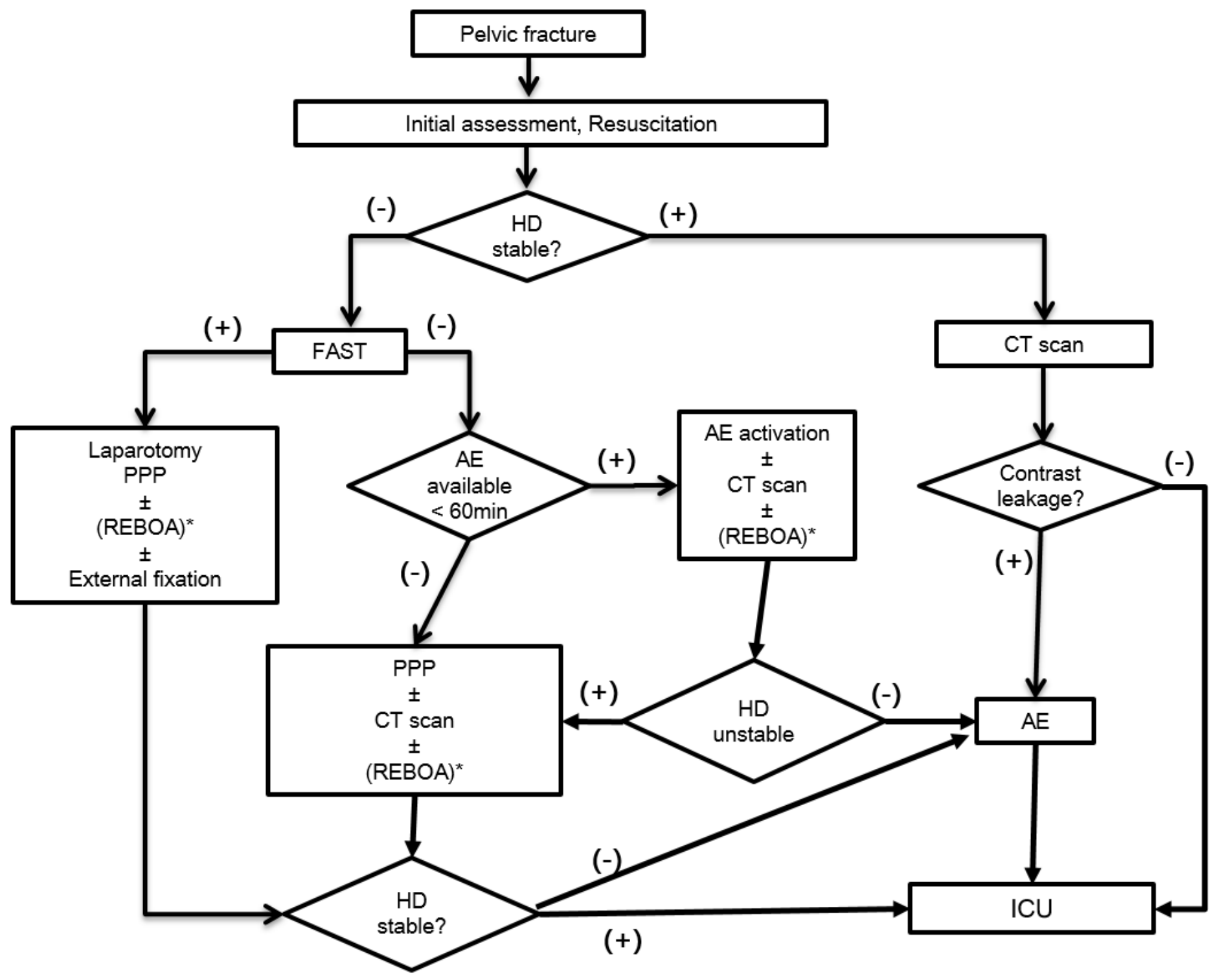

HD: hemodynamics PPP: FAST: focused assessment with sonography for trauma pre-peritoneal packing, REBOA: resuscitative endovascular balloon occlusion of aorta, $A E$ : angioembolization, ICU: intensive care unit ${ }^{*}$ REBOA was added to the algorithm in 2016

Figure 1

An algorithm for the management of pelvic fracture. 
AIS pelvis score > 1 From February 2009 to December 2018 $(\mathrm{N}=1497)$

Inclusion criteria $(\mathrm{N}=289)$

- The lowest SBP $<90 \mathrm{mmHg}$

- $\mathrm{pRBC}$ transfusion $>2$ units

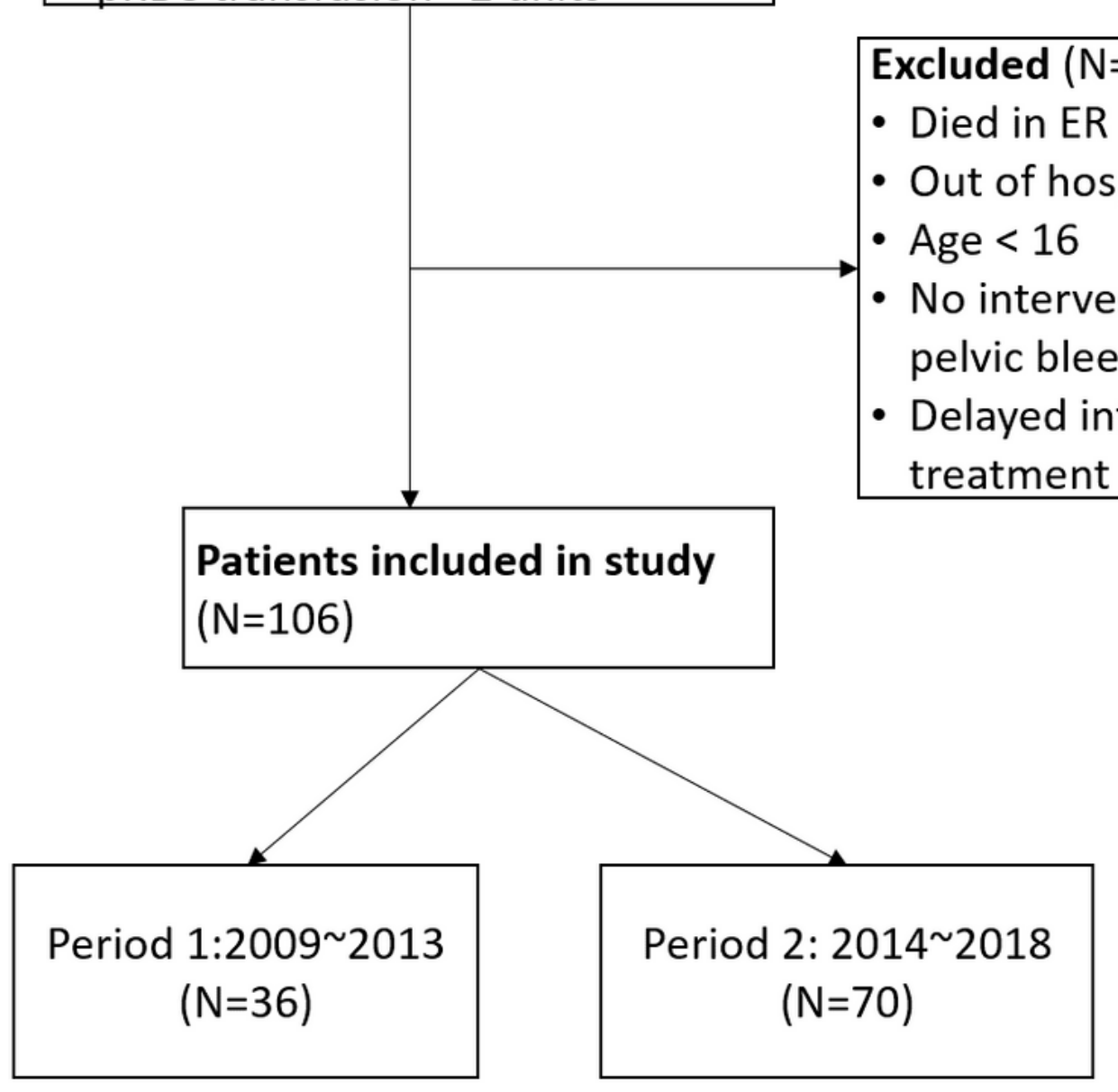

Figure 2

Flowchart of patient inclusion. 


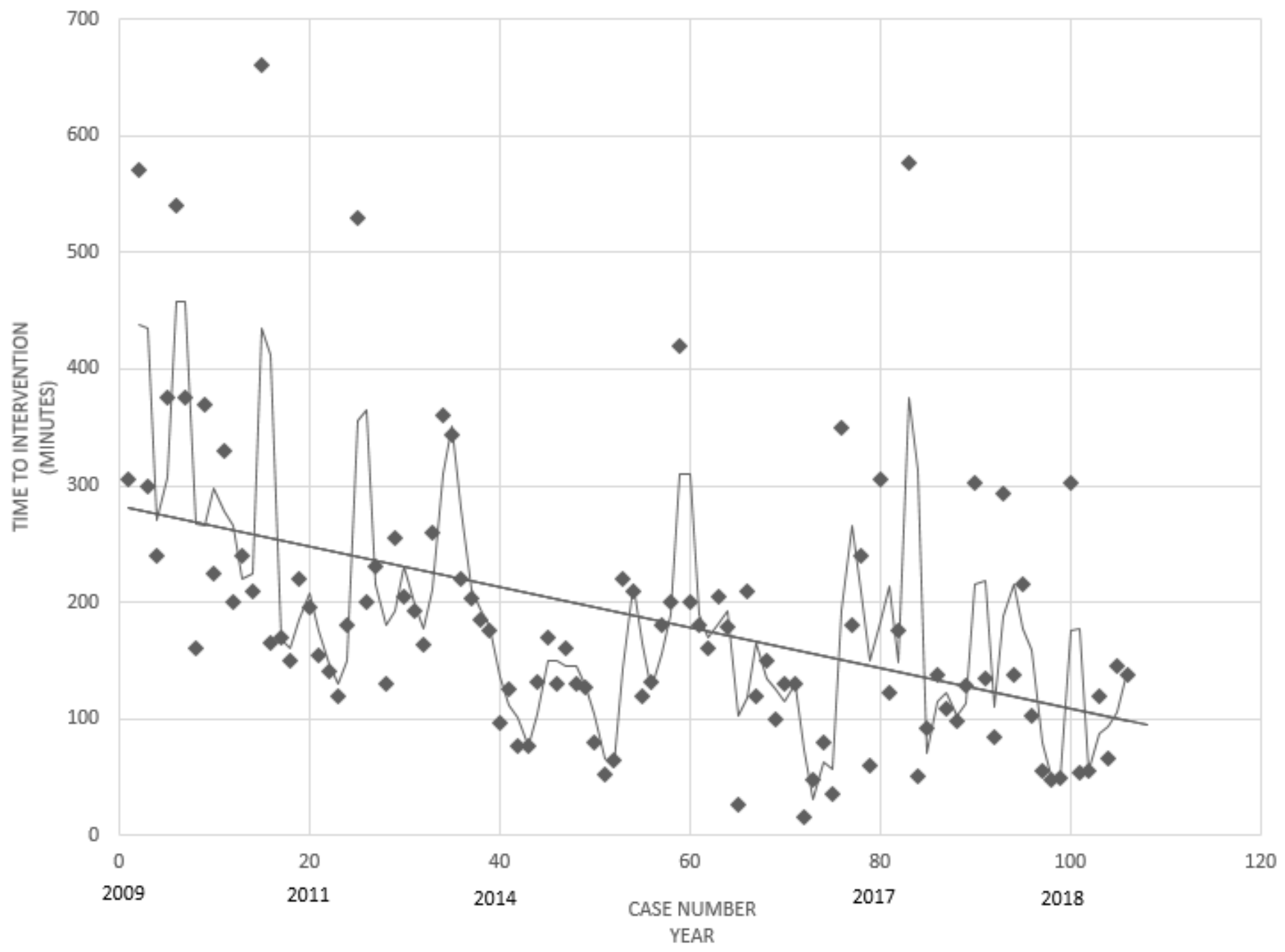

Figure 3

Time from ER to intervention throughout the study periods. 